CIENCIAMATRIA

Revista Interdisciplinaria de Humanidades, Educación, Ciencia y Tecnología

Año V. Vol. V. №1. Edición Especial. 2019

Hecho el depósito de ley: pp201602FA4721

ISSN-L: 2542-3029; ISSN: 2610-802X

Universidad Nacional Experimental Francisco de Miranda (UNEFM). Santa Ana de Coro. Venezuela

Bailon Lourido Walter Alberto

DOI 10.35381/cm.v5i1.248

\title{
Auditoria informática al control y mantenimiento de una infraestructura tecnológica
}

\section{Computer audit to control and maintenance of a technological infrastructure}

\author{
Bailon Lourido Walter Alberto \\ wbailon@hotmail.com \\ Universidad Laica Eloy Alfaro de Manabí, Manta \\ Ecuador \\ https://orcid.org/0000-0002-3262-8033
}

Recibido: 11 de agosto de 2019

Aprobado: 12 de septiembre de 2019

\begin{abstract}
RESUMEN
La investigación fue de tipo evaluativa con la finalidad de evaluar la auditoria informática al control y mantenimiento de una infraestructura tecnológica en la empresa sevimar cooperativa de la ciudad Porto Viejo - Ecuador, mediante una auditoría informática, llegándose a la conclusión de que la auditoría informática realizada, indica que la gestión de procesos informáticos y tecnológicos en la empresa investigada, no se están desarrollando eficazmente, esto implica que existe la posibilidad de generar aspectos contrarios a la conducción de una empresa basada en gestión de calidad, de acuerdo a estándares nacionales e internacionales, tanto del punto de vista organizacional como jurídico, siendo necesario que la empresa emplee medidas que favorezcan su crecimiento en el mercado desde una perspectiva de calidad total.
\end{abstract}

Descriptores: Auditoría de gestión, circulo de calidad, planificación, informática.

\begin{abstract}
The investigation was of an evaluative type with the purpose of evaluating the computer audit to the control and maintenance of a technological infrastructure in the sevimar cooperative company of the city of Porto Viejo - Ecuador, by means of a computer audit, concluding that the computer audit performed, indicates that the management of
\end{abstract}




\author{
CIENCIAMATRIA \\ Revista Interdisciplinaria de Humanidades, Educación, Ciencia y Tecnología \\ Año V. Vol. V. N¹. Edición Especial. 2019 \\ Hecho el depósito de ley: pp201602FA4721 \\ ISSN-L: 2542-3029; ISSN: 2610-802X \\ Universidad Nacional Experimental Francisco de Miranda (UNEFM). Santa Ana de Coro. Venezuela
}

\title{
Bailon Lourido Walter Alberto
}

computer and technological processes in the investigated company, are not developing effectively, this implies that there is the possibility of generating aspects contrary to the conduct of a company based on quality management, according to national and international standards, both from the organizational and legal point of view, it being necessary for the company to use measures that favor its growth in the market from a total quality perspective.

Descriptors: Management audit, Quality circles, Planning, Computer science.

\section{INTRODUCCIÓN}

Las empresas públicas y privadas requieren periódicamente de aplicar auditorías internas y externas con la finalidad de generar control sobre los diversos procesos que la conforman, esto asegura conocer cómo se desarrollan ciertos elementos, así como prevenir la mejora continua de la organización, en el actual caso de investigación, se aborda en específico a la auditoría informática, basada en la auditoria interna. La Organización Internacional de Normalización, (ISO: 2015), define la auditoría interna del siguiente modo:

Son una herramienta que ayuda a la organización a identificar posibles fallas del sistema, pero también oportunidades de mejora de los procesos internos, lo cual permite: Evaluar la eficacia de los controles internos. Contribuir al proceso de mejoría de los procesos y del Sistema en general. Comprobar y monitorear el cumplimiento de las normas y los procedimientos vigentes. Analizar la aparición de nuevos riesgos, permitiendo la implementación de procedimientos para minimizar o neutralizar su impacto.

Así mismo esta aplicabilidad ha sido trasladada a los sistemas informáticos y computarizados de la empresa, así la ISO, genera la norma ISO-27001, la cual describe cómo la gestión de la seguridad de la información en una empresa. Kosutic (2017) señala que "la filosofía principal de la norma ISO 27001 se basa en la gestión de riesgos: investigar dónde están los riesgos y luego tratarlos sistemáticamente". Esto implica la necesidad de promover en las empresas una auditoría focalizada a los procesos tecnológicos, con la finalidad de prever a tiempo como se está desarrollando en un marco de calidad. Kosutic (2017), describe que "hay 4 ventajas comerciales esenciales que una 


\section{Bailon Lourido Walter Alberto}

empresa puede obtener con la implementación de esta norma para la seguridad de la información:

1. Cumplir con los requerimientos legales - cada vez hay más y más leyes, normativas y requerimientos contractuales relacionados con la seguridad de la información. La buena noticia es que la mayoría de ellos se pueden resolver implementando ISO 27001 ya que esta norma le proporciona una metodología perfecta para cumplir con todos ellos.

2. Obtener una ventaja comercial - si su empresa obtiene la certificación y sus competidores no, es posible que usted obtenga una ventaja sobre ellos ante los ojos de los clientes a los que les interesa mantener en forma segura su información.

3. Menores costos - la filosofía principal de ISO 27001 es evitar que se produzcan incidentes de seguridad, y cada incidente, ya sea grande o pequeño, cuesta dinero; por lo tanto, evitándolos su empresa va a ahorrar mucho dinero. Y lo mejor de todo es que la inversión en ISO 27001 es mucho menor que el ahorro que obtendrá.

4. Una mejor organización - en general, las empresas de rápido crecimiento no tienen tiempo para hacer una pausa y definir sus procesos y procedimientos; como consecuencia, muchas veces los empleados no saben qué hay que hacer, cuándo y quién debe hacerlo. La implementación de ISO 27001 ayuda a resolver este tipo de situaciones ya que alienta a las empresas a escribir sus principales procesos (incluso los que no están relacionados con la seguridad), lo que les permite reducir el tiempo perdido de sus empleados".

La norma ISO-27001 proyecta mejorar el rendimiento efectivo y productivo de la empresa, permite evaluar sobre la marcha sin tener en cuenta retrasos, promoviendo mayor pertinencia en los empleados en lograr en obtener un mejor rendimiento en la medida que conocen las debilidades del sistema, aunado a lo planteado, es de destacar que la ISO trabaja en la gestión por procesos, mediante la aplicación del ciclo: Planificar, Hacer, 


\section{Bailon Lourido Walter Alberto}

Verificar, Actuar, conocido por sus siglas "PHVA", de ese modo, la ISO 9001 (2015) señala que este se basa en "un enfoque global de pensamiento basado en riesgos", así mismo, plantea que "la aplicación del enfoque a procesos en un sistema de gestión de la calidad permite:
a) La comprensión y el cumplimiento de los requisitos de manera coherente;
b) La consideración de los procesos en términos de valor agregado;
c) El logro de un desempeño del proceso eficaz;
d) La mejora de los procesos con base en la evaluación de los datos y la información (p. 8).

Lo cual posibilita a las empresas trabajar desde la perspectiva de la mejora continua, por consiguiente de audita con la finalidad de crecer permanentemente en función de: Planificar, Hacer, Verificar, Actuar, (PHVA), siendo conocido como el ciclo PHVA:

1. Planificar: establecer los objetivos del sistema y sus procesos, y los recursos necesarios para generar y proporcionar resultados de acuerdo con los requisitos del cliente y las políticas de la organización, e identificar y abordar los riesgos y las oportunidades;

2. Hacer: implementar lo planificado;

3. Verificar: realizar el seguimiento y (cuando sea aplicable) la medición de los procesos y los productos y servicios resultantes respecto a las políticas, los objetivos, los requisitos y las-actividades planificadas, e informar sobre los resultados;

4. Actuar; tomar acciones para mejorar el desempeño, cuando sea necesario (p. 10). Desde lo planteado se busca una gestión de calidad en beneficio del cliente, lo cual permite a la organización focalizar servicios que satisfagan las expectativas del cliente, Aldana \& Piña (2017), señalan que esto permite a la organización obtener fidelidad de los clientes internos y externos de la organización, esto conlleva a tener una visión de procesos de mejora continua, en este sentido, la ISO 9001: 2015, indica que gestión por procesos se basa en: 


\section{Bailon Lourido Walter Alberto}

La comprensión y gestión de los procesos interrelacionados como un sistema contribuye a la eficacia y eficiencia de la organización en el logro de sus resultados previstos. Este enfoque permite a la organización controlar las interrelaciones e interdependencias entre los procesos del sistema, de modo que se pueda mejorar el desempeño global de la organización.

El generar una auditoría desde el enfoque de procesos y de la norma ISO 27001, contribuye a fortalecer los procesos de calidad en la empresa, aunado a que esta es una normativa de la contraloría general de la República del Ecuador, lo cual fomenta una triple responsabilidad para las organizaciones que permiten cumplir con procesos de gestión de calidad y legal, siendo esto importante porque a las empresas del Ecuador, le permite estar en consonancia con una gerencia pública focalizada a promover servicios de calidad, basado en auditar internamente sus componentes tecnológicos e informáticos, siendo estos pilares esenciales en la articulación de políticas basadas en la generación de gestión por procesos, en tal sentido, la investigación tuvo por objetivo evaluar la auditoria informática al control y mantenimiento de una infraestructura tecnológica en la empresa sevimar cooperativa de la ciudad Porto Viejo - Ecuador.

\section{DESARROLLO}

\section{Auditoria informática}

En este sentido, Piattini, et al. (2008), plantea que la auditoría en informática se despliega en función de promover una evaluación en función de estándares nacionales e internacionales, por otro lado, Ramírez \& Álvarez (2003), destaca la importancia de abordar áreas en la generación de una auditoría informática, entre las cuales se encuentran:

1. Administración de los recursos de TI: Para asegurar que la Entidad utilice tecnología de información bajo criterios de costo-beneficio, considerando las necesidades de automatización y adecuación de cambios del entorno en que se desenvuelve. 


\section{Bailon Lourido Walter Alberto}

2. Seguridad Física y Seguridad de Información: Para garantizar que el ambiente en el que los sistemas funcionan protege su confidencialidad, integridad y confiabilidad, la reducción al mínimo del riesgo de que ocurran daños accidentales o intencionales a los equipos.

3. Desarrollo y Mantenimiento de Sistemas de Información de la Entidad: Para garantizar la disponibilidad de los sistemas cuando se necesiten, que se controle la integridad de los datos y que satisfagan a los usuarios.

4. Continuidad de SI de la Entidad: Reducir al mínimo la posibilidad de que ocurra un desastre total y garantizar que el negocio pueda reanudar sus operaciones con efectividad en caso de que ya no se disponga de las instalaciones de procesamiento existentes.

De ese modo, se genera la posibilidad de promover una gestión de procesos que pueda ser auditada de modo integral, focalizándose en las áreas referentes a la informática, siendo indispensable conocer cómo se generan estos procesos, para lo cual, la auditoría toma un rol secundario de prever acciones mediante un proceso continuo de revisión, teniendo como relación directa con la gestión de procesos de Planificar, Hacer, Verificar, Actuar, (PHVA), para lo cual se requiere que los auditores cuenten con los manuales o protocolos de gestión inherentes a la organización, situación que permitirá verificar correctamente el desempeño informático y tecnológico de la empresa con la finalidad de intervenir a tiempo en función de mantener o elevar la calidad.

\section{Pasos para realizar una auditoría informática}

En este renglón la organización Tendencias \& Innovación (2018), plantea que es importante que conozcamos los pasos previos que debemos seguir para realizar una auditoría aunque sea un experto quien la realice:

1. Análisis para poder establecer cuáles son los objetivos que tiene la empresa, hacia dónde quiere llegar y así tener capacidad de conocer más adelante la eficacia que presenta su sistema informático atendiendo a dichos objetivos. 


\section{Bailon Lourido Walter Alberto}

2. Un inventario con todos los puntos elaborados. Este inventario va a ser el protagonista en el siguiente paso, ya que nos servirá para llevar a cabo una planificación en la cual debemos fijarnos en cómo vamos a estudiar cada uno de esos puntos para que siempre ofrezcan el mejor rendimiento posible.

3. Delimitar cuáles son las incidencias que nos hemos encontrado a lo largo de todo el análisis y los riesgos a los que nuestro sistema informático está expuesto para no realizar su trabajo de forma correcta.

4. Por último, poner en marcha todas las técnicas y métodos necesarios para resolver los problemas que hayan ido apareciendo durante el análisis del sistema informático y así poder dar una solución prácticamente inmediata.

El auditor debe contar con los insumos necesarios que le permitan conocer e identificar las fallas y potencialidades del sistema a auditar, esto complementa la posibilidad de lograr un proceso de calidad en función de generar eficacia y transparencia en los procesos auditores de la empresa, así mismo contribuye en que la empresa genere un control basado en gestión de calidad, tomando correctivos sobre la marcha para estar en sintonía de crecer continuamente.

\section{Normas de control interno para el área de sistemas de información computarizados}

En este sentido, la contraloría general del Ecuador, prevé los siguientes aspectos:

\section{Utilización de los equipos, programas e información institucional}

\section{Normas de control interno para el área de sistemas de información computarizados}

- Con estas normas se busca promover la correcta utilización de los sistemas computarizados que procesan la información que generan las entidades.

- La máxima autoridad de cada entidad pública o por su delegación los directivos y jefes de las unidades administrativas, establecerán procedimientos para asegurar el uso eficiente, eficaz y económico de los equipos, programas de computación e información computarizada, a través de: 


\section{Bailon Lourido Walter Alberto}

1. El registro y seguimiento de la operación de los mismos;

2. La capacitación de funcionarios en la utilización de los equipos y programas; y,

3. La evaluación periódica de los objetivos cumplidos mediante la computarización.

Los equipos y programas serán utilizados exclusivamente en las actividades propias de la institución. La información obtenida del proceso computarizado será de uso e intercambio entre las instituciones del sector público, con excepción de aquella considerada expresamente como reservada o de uso restringido. En el caso de fijarse un valor para la información a proporcionar a estas entidades, el mismo considerará únicamente los costos de su obtención, procesamiento y transmisión que será materia del reglamento pertinente.

\section{Administración del software}

- Dado que el software, está protegido por la Ley de Derecho de Autor y no puede utilizarse, reproducirse o distribuirse sin la autorización expresa del fabricante; con la finalidad de administrar, garantizar la legitimidad del software para evitar disputas legales a futuro, es necesario formular políticas institucionales del software, que cubra la adquisición y el uso del software en las entidades del Sector Público.

Son algunas de las normativas vigente por la contraloría del Ecuador en función de auditar lo relacionado a sistemas computarizados, siendo necesario aplicar en conjunto a la gestión de procesos y normas de la ISO, con la finalidad de validar bajo criterios estandarizados, el accionar los procesos informáticos - tecnológicos de las empresas en razón de proyectar una organización en base a la calidad, tal como propone Huapaya Capcha (2019).

\section{METODOLOGÍA}

La investigación fue de tipo evaluativa, Hurtado (2006), con la finalidad de evaluar la auditoria informática al control y mantenimiento de una infraestructura tecnológica en la 


\section{Bailon Lourido Walter Alberto}

empresa sevimar cooperativa de la ciudad Porto Viejo - Ecuador, de ese modo, se procedió a establecer un sistema de rango que permitió conocer el rango de ubicación y así conocer el nivel en el cual se encuentran los procesos de auditoría informática, la cual fue realizada al departamento de informática de la empresa objeto de estudio, siendo la población 6 personas que conforman el talento humano de la mencionada institución.

\section{DISCUSIÓN DE LOS RESULTADOS}

\section{Cuadro 1}

Administración de los recursos de TI

\begin{tabular}{|c|c|c|c|c|c|c|}
\hline \multirow{4}{*}{$\begin{array}{c}\text { Respuestas } \\
\text { Deficiente } \\
\text { eficiencia en } \\
\text { auditoria } \\
\text { informática } \\
\text { Regular eficiencia } \\
\text { en auditoria } \\
\text { informática } \\
\text { Excelente } \\
\text { eficiencia en } \\
\text { auditoria } \\
\text { informática }\end{array}$} & \multirow{2}{*}{$\begin{array}{c}\text { Rango } \\
\geq 0< \\
0,666\end{array}$} & \multirow{2}{*}{$\begin{array}{l}\text { Categoría } \\
\text { Deficiente }\end{array}$} & \multicolumn{2}{|c|}{ Estadístico } & $\mathrm{Fa}$ & $\%$ \\
\hline & & & $\begin{array}{l}\text { Media } \\
\text { aritmética }\end{array}$ & 0,4 & 4 & 67 \\
\hline & $\begin{array}{c}\geq 0,666< \\
1,332\end{array}$ & Regular & Mediana & 0 & 2 & 33 \\
\hline & $\begin{array}{c}\geq 1,332< \\
1,998\end{array}$ & Excelente & Moda & 0 & 0 & 0 \\
\hline & & & $\begin{array}{c}\text { Desviación } \\
\text { Estándar }\end{array}$ & 0,4 & & \\
\hline
\end{tabular}

La media obtenida indica que la administración de recursos $\mathrm{TI}$, se encuentra en una categoría deficiente, lo cual discrepa de la visión de Ramírez \& Álvarez (2003), aunado que contradice los principios de gestión por procesos y de calidad establecidos por la ISO.

Por lo tanto, no se evidencia que la empresa implemente tecnología que le beneficie en relación costo - beneficio, lo cual conlleva a pensar en la posibilidad de que se está 
invirtiendo en productos de capacidad que no se corresponden a las necesidades reales de la empresa, de ese modo, no se genera el aprovechamiento de los recursos que ofrece el mercado hoy día, por cuanto se ofertan productos informativos y tecnológicos en posibilidad de ampliar la eficiencia, rentabilidad, en concordancia con invertir en productos de mayor vanguardia, como de menor precio, siendo necesaria la inversión de nuevos proyectos informáticos y tecnológicos en pro de favorecer la productividad de la empresa.

\section{Cuadro 2}

Seguridad Física y Seguridad de Información

\begin{tabular}{|c|c|c|c|c|c|c|}
\hline Respuestas & Rango & Categoría & \multicolumn{2}{|c|}{ Estadístico } & $\mathrm{Fa}$ & $\%$ \\
\hline $\begin{array}{c}\text { Deficiente } \\
\text { eficiencia en } \\
\text { auditoria } \\
\text { informática }\end{array}$ & $\begin{array}{l}\geq 0< \\
0,666\end{array}$ & Deficiente & $\begin{array}{l}\text { Media } \\
\text { aritmética }\end{array}$ & 0,5 & 4 & 83 \\
\hline $\begin{array}{l}\text { Regular eficiencia } \\
\text { en auditoria } \\
\text { informática }\end{array}$ & $\begin{array}{c}\geq 0,666< \\
1,332\end{array}$ & Regular & Mediana & 0 & 2 & 17 \\
\hline $\begin{array}{l}\text { Excelente } \\
\text { eficiencia en } \\
\text { auditoria } \\
\text { informática }\end{array}$ & $\begin{array}{c}\geq 1,332< \\
1,998\end{array}$ & Excelente & Moda & 0 & 0 & 0 \\
\hline
\end{tabular}

TOTAL 31

La media obtenida indica que la seguridad física y seguridad de información se encuentra en una categoría deficiente, lo cual discrepa de la visión de Ramírez \& Álvarez (2003), aunado que contradice los principios de gestión por procesos y de calidad establecidos por la ISO.

De acuerdo al autor precitado, la seguridad física y seguridad de información, permite "garantizar que el ambiente en el que los sistemas funcionan protege su confidencialidad, 
integridad y confiabilidad, la reducción al mínimo del riesgo de que ocurran daños accidentales o intencionales a los equipos". De ese modo, se percibe que la seguridad informática y tecnológica no se está implementando acordemente a lo establecido en la teoría, ocasionando riesgos en la seguridad que puede interferir en la pérdida de información tanto para la empresa como para sus clientes, siendo necesaria implementar medidas de seguridad conforme a postulados que permitan promover eficiencia en la consecución de promover una organización que permita trabajar con eficacia en la medida que proyecta seguridad, siendo esto un pilar fundamental para concretar la fidelidad del cliente externo.

\section{Cuadro 3}

\section{Desarrollo y mantenimiento de sistemas de información de la entidad}

\begin{tabular}{|c|c|c|c|c|c|c|}
\hline Respuestas & Rango & Categoría & \multicolumn{2}{|c|}{ Estadístico } & $\mathrm{Fa}$ & $\%$ \\
\hline $\begin{array}{c}\text { Deficiente } \\
\text { eficiencia en } \\
\text { auditoria } \\
\text { informática }\end{array}$ & $\begin{array}{l}\geq 0< \\
0,666\end{array}$ & Deficiente & $\begin{array}{l}\text { Media } \\
\text { aritmética }\end{array}$ & 0 & 2 & 35 \\
\hline $\begin{array}{l}\text { Regular eficiencia } \\
\text { en auditoria } \\
\text { informática }\end{array}$ & $\begin{array}{c}\geq 0,666< \\
1,332\end{array}$ & Regular & Mediana & 0,7 & 4 & 65 \\
\hline $\begin{array}{l}\text { Excelente } \\
\text { eficiencia en } \\
\text { auditoria }\end{array}$ & $\begin{array}{c}\geq 1,332< \\
1,998\end{array}$ & Excelente & Moda & 0 & 0 & 0 \\
\hline
\end{tabular}

\begin{tabular}{cccc}
$\begin{array}{c}\text { Desviación } \\
\text { Estándar }\end{array}$ & 0,7 & \\
\hline TOTAL & 31 & 100
\end{tabular}

La media obtenida indica que el desarrollo y mantenimiento de sistemas de información de la entidad, se encuentra en una categoría regular, lo concuerda regularmente con la visión de Ramírez \& Álvarez (2003), lo cual implica que es necesario mejorar para estar 
en concordancia con los principios de gestión por procesos y de calidad establecidos por la ISO.

Así la empresa tendrá mayor oportunidad de plantearse una gestión por procesos de calidad en base al mantenimiento efectivo que realiza permanente, por cuanto se hace necesario que la empresa regularice en función de generar un proceso de mejora continua en su sistema tecnológico e informático, lo cual permitirá establecer un servicio de calidad de forma sistemática, evitando que colapsen los servicios informáticos, siendo estos pilares en la generación de información al cliente, de ese modo, establecer un principio de mantenimiento, genera además cultura organizacional basada en el crecimiento permanente de la empresa con fines de ampliar su capacidad de oferta en el mercado.

\section{Cuadro 4}

Continuidad de SI de la entidad

\begin{tabular}{|c|c|c|c|c|c|c|}
\hline Respuestas & Rango & Categoría & \multicolumn{2}{|c|}{ Estadístico } & $\mathrm{Fa}$ & $\%$ \\
\hline $\begin{array}{c}\text { Deficiente } \\
\text { eficiencia en } \\
\text { auditoria } \\
\text { informática }\end{array}$ & $\begin{array}{l}\geq 0< \\
0,666\end{array}$ & Deficiente & $\begin{array}{l}\text { Media } \\
\text { aritmética }\end{array}$ & 0 & 2 & 35 \\
\hline $\begin{array}{l}\text { Regular eficiencia } \\
\text { en auditoria } \\
\text { informática }\end{array}$ & $\begin{array}{c}\geq 0,666< \\
1,332\end{array}$ & Regular & Mediana & 0,7 & 4 & 65 \\
\hline $\begin{array}{l}\text { Excelente } \\
\text { eficiencia en } \\
\text { auditoria }\end{array}$ & $\begin{array}{c}\geq 1,332< \\
1,998\end{array}$ & Excelente & Moda & 0 & 0 & 0 \\
\hline & & & $\begin{array}{l}\text { Desviación } \\
\text { Estándar }\end{array}$ & 0,7 & & \\
\hline
\end{tabular}

TOTAL 31 


\section{Bailon Lourido Walter Alberto}

La media obtenida indica que la continuidad de SI de la entidad, se encuentra en una categoría regular, lo concuerda regularmente con la visión de Ramírez \& Álvarez (2003), lo cual implica que es necesario mejorar para estar en concordancia con los principios de gestión por procesos y de calidad establecidos por la ISO.

Este renglón permite auditar la posibilidad de que ocurra un evento catastrófico en el sistema tecnológico de la empresa, lo cual imposibilitaría su operación de modo significativo y eficaz, siendo pertinente realizar continuamente evaluaciones que permitan focalizar posibles problemas en función de evitar la paralización de los servicios de la empresa, por cuanto esto solo generaría pérdidas proporcionales en relación de ocasionar el deterioro de la de fidelidad de la cliente externa, originando una imagen corporativa negativa.

\section{CONCLUSIONES}

La auditoría informática realizada, indica que la gestión de procesos informáticos y tecnológicos en la empresa investigada, no se están desarrollando eficazmente, esto implica que existe la posibilidad de generar aspectos contrarios a la conducción de una empresa basada en gestión de calidad, de acuerdo a estándares nacionales e internacionales, tanto del punto de vista organizacional como jurídico, siendo necesario que la empresa emplee medidas que favorezcan su crecimiento en el mercado desde una perspectiva de calidad total.

La auditoría informática tiene un rol fundamental en la empresa al igual que la auditoría interna, de ese modo, no puede menospreciarse la generación de auditorías con fines informáticos y tecnológicos, más aun cuando las empresas modernas dependen en gran parte de generar operaciones bajo estos sistemas, por lo tanto una eficiente auditoria informática, contribuye además en evaluar para aplicar la mejorar continua en función de lo planteado por la ISO, en este sentido, se puede aplicar este tipo de investigaciones a otras empresas en donde se cuenten con programas informáticos y tecnológicos, 


\section{Bailon Lourido Walter Alberto}

generándose progresivamente la cultura de gestión evaluativa en función de lo auditable desde la informática.

\section{REFERENCIAS CONSULTADAS}

1. Aldana, J., \& Piña, J. (2017). Calidad del servicio prestado al cliente por los instructores de gimnasios. Revista Arbitrada Interdisciplinaria Koinonía, 2(3), 172197. Recuperado http://fundacionkoinonia.com.ve/ojs/index.php/revistakoinonia/article/view/59/46

2. Contraloría general del Ecuador (2016). Normas de control interno para el sector público. Recuperado de https://www.cancilleria.gob.ec/wpcontent/uploads/downloads/2013/02/normas_control_int.pdf

3. Hurtado, de Barrera, J. (2006). El proyecto de investigación. Metodología de la investigación holística. Ediciones SYPAL. Bogotá. Cuarta Edición.

4. Huapaya Capcha, Y. (2019). Gestión por procesos hacia la calidad educativa en el Perú. Revista Arbitrada Interdisciplinaria Koinonía, 4(8), 243-261. doi:http://dx.doi.org/10.35381/r.k.v4i8.277

5. Kosutic, D. (2017) ¿Qué es norma ISO 27001? Recuperado de https://advisera.com/27001academy/es/que-es-iso-27001/

6. Ramírez R., Guadalupe, \& Álvarez D., Ezzard (2003). Auditoría a la Gestión de las Tecnologías y Sistemas de Información. Recuperado de https://www.redalyc.org/pdf/816/81606114.pdf

7. Tendencias \& Innovación (2018). Pasos para realizar una auditoría informática. Recuperado de https://www.obs-edu.com/int/blog-investigacion/sistemas/quees-una-auditoria-informatica-y-que-debes-saber-sobre-ella

8. Piattini, M; Del Peso, E.; Del Peso, M. 2008. Auditoria de Tecnologías y Sistemas de Información. 4ed. Madrid, ES. RA-MAI. Vol. 1378. p 38. 
CIENCIAMATRIA

Revista Interdisciplinaria de Humanidades, Educación, Ciencia y Tecnología

Año V. Vol. V. NN1. Edición Especial. 2019

Hecho el depósito de ley: pp201602FA4721

ISSN-L: 2542-3029; ISSN: 2610-802X

Universidad Nacional Experimental Francisco de Miranda (UNEFM). Santa Ana de Coro. Venezuela

\section{Bailon Lourido Walter Alberto}

\section{REFERENCES CONSULTED}

1. Aldana, J., \& Piña, J. (2017). Quality of service provided to the client by gym instructors. Interdisciplinary Arbitrated Review Koinonía, 2 (3), 172-197. Recovered from

http://fundacionkoinonia.com.ve/ojs/index.php/revistakoinonia/article/view/59/46

2. Comptroller General of Ecuador (2016). Internal control standards for the public sector. Recovered from https://www.cancilleria.gob.ec/wpcontent/uploads/downloads/2013/02/normas control int.pdf

3. Hurtado, de Barrera, J. (2006). The research project Holistic research methodology. SYPAL editions. Bogota Fourth edition.

4. Huapaya Capcha, Y. (2019). Management by processes towards educational quality in Peru. Interdisciplinary Arbitrated Review Koinonía, 4 (8), 243-261. doi: http: //dx.doi.org/10.35381/r.k.v4i8.277

5. Kosutic, D. (2017) What is ISO 27001? Recovered from https://advisera.com/27001academy/es/que-es-iso-27001/

6. Ramírez R., Guadalupe, \& Álvarez D., Ezzard (2003). Audit to the Management of Technologies and Information Systems. Recovered from https://www.redalyc.org/pdf/816/81606114.pdf

7. Trends \& Innovation (2018). Steps to perform a computer audit. Recovered from https://www.obs-edu.com/int/blog-investigacion/sistemas/que-es-una-auditoriainformatica-y-que-debes-saber-sobre-ella

8. Piattini, M; Of the Weight, E .; Del Peso, M. 2008. Audit of Technologies and Information Systems. 4ed. Madrid, ES. Branch. Vol. 1378. p 38.

(C2019 por los autores. Este artículo es de acceso abierto y distribuido según los términos y condiciones de la licencia Creative Commons Atribución-NoComercial-Compartirlgual 4.0 Internacional (CC BY-NC-

SA 4.0) (https://creativecommons.org/licenses/by-nc-sa/4.0/). 